\title{
HOMOLOGICAL GEOMETRY I. PROJECTIVE HYPERSURFACES
}

\author{
Alexander B. GIVENTAL \\ Dept. of Math., UC Berkeley \\ Berkeley CA 94720, USA *
}

May 24, Revised August 15, 1994

\section{Introduction}

Consider a generic quintic hypersurface $X$ in $\mathbb{C} P^{4}$. It is an example of CalabiYau 3-folds. It follows from Riemann-Roch formula, that rational curves on a generic Calabi-Yau 3-fold should be situated in a discrete fashion. Therefore a natural question of enumerative algebraic geometry arises: find the number $n_{d}$ of rational curves in $X$ of degree $d$ for each $d=1,2,3, \ldots$ In 1991 Candelas, de la Ossa, Green and Parkes [1] 'predicted' all the numbers $n_{d}$ simultaneously: they conjectured that the generating function

$$
K(Q)=5+\sum_{d=1}^{\infty} \frac{n_{d} d^{3} Q^{d}}{1-Q^{d}}
$$

can be found by studying the 4-th order linear differential operator annihilating some hypergeometric series, namely

$$
\sum_{d=0}^{\infty} \frac{(5 d) ! q^{d}}{(d !)^{5}}
$$

*This research is partially supported by Alfred P. Sloan Foundation and by NSF Grant DMS-9321915. 
The conjecture was motivated by some ideas of conformal topological field theory (CTFT): there is a 1-parametric family $Y_{q}$ of Calabi-Yau 3-folds which are 'mirrors' of the quintics $X$ in the sense that their Hodge numbers satisfy $h^{r, s}(Y)=h^{3-r, s}(X)$. This indicates that a model of CTFT dealing with rational curves in $X$ might be equivalent to a model based on periods of holomorphic 3-forms in $Y_{q}$. Therefore problems about rational curves in $X$ can be transformed into those about the Picard-Fuchs equation for periods of holomorphic forms in $Y$. The hypergeometric series in question is in fact one of such periods.

The 'mirror conjecture' about equivalence of algebraic geometry of rational curves in Calabi-Yau manifolds and variations of Hodge structures on their mirrors, as well as an explicit construction of such mirror pairs, has been generalized to a broad class of Calabi-Yau complete intersections in toric varieties (see Batyrev [2] and Batyrev - Van-Straten [3]) and supported by numerous verified corollaries and 'experimental' data (see papers by D.Morrison and references therein). However the mirror phenomenon itself, and especially the relation between rational curves in $X$ and the PicardFuchs differential equation for $Y$ remain mysterious.

In this paper, we make a step toward explanation of the mirror conjecture. Namely we describe how hypergeometric differential equations arise in connection with algebraic geometry of rational curves in Kahler manifolds. In particular, the differential equations which one used to observe in the theory of mirror manifolds as Picard-Fichs equations for periods of holomorphic forms "on $Y$ ", will be obtained naturally in terms of geometry of rational curves in $X$.

In Section 1 we remind some basic facts about equivariant cohomology and formulate a simple lemma which already contains a germ of mirror phenomena. In Section 2 we describe a project called Equivariant Floer cohomology which is supposed to explain the role of differential equations in algebraic geometry of rational curves: we show that the equivariant Floer cohomology should bear a $D$-module structure. While it may be not easy to make the whole project rigorous, one can make its idea work in some examples, namely in the case of toric manifolds and toric complete intersections. For the sake of simplicity we concentrate here on projective hypersurfaces and derive in Sections 3 and 4 complete solutions of certain hypergeometric differential equations written down in cohomological terms of spaces of maps $\mathbb{C} P^{1} \rightarrow \mathbb{C} P^{n-1}$. More general case of complete intersections in toric 
manifolds can be treated analogously (see [15]). In a forthcoming paper we will explain why the differential equations in question are Picard-Fuchs i. e. we will give a natural integral representation for their solutions - also in cohomological terms.

Applying our constructions to the problem about numbers of rational curves on quintics, one should keep in mind the 'strange' computational procedure used in [1] and [3]:

Consider the linear differential operator

$$
D^{4}-5 q(5 D+1)(5 D+2)(5 D+3)(5 D+4) \text { where } D=q \frac{d}{d q} \text {. }
$$

It has a singularity at $q=0$ and $q=1 / 5^{5}$ and the monodromy of solutions at $q=0$ is known to be unipotent. In particular this means that there is a single-valued solution $f_{0}(q)$ (it is given by the hypergeometric series above) and a solution of the form $\log (q) f_{0}(q)+f_{1}(q)$ where $f_{1}$ is holomorphic near $q=0$ and $f_{1}(0)=0$. Introduce then a new local coordinate

$$
Q(q)=q \exp \left(f_{1}(q) / f_{0}(q)\right)
$$

Take the 'renormalized Yukawa 3-differential'

$$
\frac{5}{\left(1-5^{5} q\right) f_{0}(q)^{2}}\left(\frac{d q}{q}\right)^{\otimes 3}
$$

and transform it to the new local coordinate $Q$ so that it assumes the form $k(Q)(d Q / Q)^{\otimes 3}$. Then the mirror conjecture for quintics says that the function $k(Q)$ coincides with the generating function $K(Q)$ for the numbers $n_{d}$ of rational curves of degree $d$.

Our results reduce this conjecture to the statement (formulated with more detail in Section 5) that two different problems, both about rational curves on quintics, lead to two different 4-th order linear ODE whose 4 solutions, however, describe proportional unparametrized curves in $\mathbb{C}^{4}$. From this point of view the above 'strange' computational procedure becomes rather natural.

An observation crucial for this paper was made during my visit to UNC at Chapel Hill. I am thankful to all members of the Department of Mathematics and especially to my host A.Varchenko for hospitality and stimulating discussions. I would like also to thank Institute Henri Poincare where this paper is being completed. 


\section{Equivariant cohomology}

We begin with a brief discussion of our principal tool in this paper - equivariant cohomology of hamiltonian circle actions.

Let $M$ be a topological space provided with a compact group $G$ action, let $E G \rightarrow B G$ be the universal principal $G$-bundle. Equivariant cohomology $H_{G}^{*}(M)$ is defined as $H^{*}\left(M_{G}\right)$ where $M_{G}=E G \times{ }_{G} M=(E G \times M) / G$ is the homotopic quotient of $M$ by $G$. The projection $M_{G} \rightarrow B G=E G / G=(p t)_{G}$ provides $H_{G}^{*}(M)$ with a module structure over the characteristic class ring $H_{G}^{*}(p t)=H^{*}(B G)$.

Essentially we need the case where $M$ is a compact complex manifold provided with a holomorphic action of the complex circle $\mathbb{C}-0$ containing $S^{1}$. Then $E S^{1}$ can be identified with $\mathbb{C}^{\infty}-0$ or the unit sphere therein, and the classifying space $B S^{1}$ - with $\mathbb{C} P^{\infty}$. One can also use finite-dimensional approximations of the universal bundle by the Hopf bundle $\left(\mathbb{C}^{N}-0\right) /(\mathbb{C}-0)=$ $\mathbb{C} P^{N-1}$ and define equivariant cohomology as a limit. Setting the convention that all cohomologies are with complex coefficients we describe the coefficient ring of the $S^{1}$-equivariant theory as a polynomial algebra with one generator of degree 2 (represented by hyperplane sections in $\mathbb{C} P^{\infty}$ ). We will see soon that the natural notation for this generator is $\hbar$ :

$$
H_{S^{1}}^{*}(p t)=H^{*}\left(\mathbb{C} P^{\infty}\right)=\mathbb{C}[\hbar] .
$$

In the case when $M$ is a compact manifold one can define the push forward operation $H_{G}^{*}(M) \rightarrow H_{G}^{*}(p t)$ which consists in fiberwise integration in the bundle $M_{G} \rightarrow B G$ along the fibers $M$.

There is also a De Rham model for equivariant cohomology of a manifold $M$. The $S^{1}$-equivariant De Rham complex consists of differential forms on $M$ depending in a polynomial way on the parameter $\hbar$ and invariant with respect to the circle action on $M$. If $v$ denotes the vector field generating the action then the equivariant De Rham differential is $d+\hbar i_{v}$. Cohomolohy of such complex is known to coincide with the equivariant cohomology defined above.

This construction was exploited by Atiyah-Bott [4] and Berline-Vergne [14] in order to explain Duistermaat - Heckman formula for hamiltonian circle actions. Let $M$ be a compact symplectic $2 m$-dimensional manifold, $\omega$ - the symplectic form, $H$ - the Hamilton function of the circle action, $F_{\alpha}$ 
— critical manifolds of $H$ (= the fixed point sets of the action) with critical values $H_{\alpha}$. The formula says that

$$
\int_{M} e^{\hbar H} \frac{\omega^{m}}{m !}=\sum_{\alpha} e^{\hbar H_{\alpha}} \int_{F_{\alpha}} \frac{e^{\omega}}{E_{\alpha}}
$$

where $E_{\alpha}$ is the equivariant Euler class of the normal bundle to $F_{\alpha}$ in $M$.

The RHS of the Duistermaat-Heckman formula is in fact the principal term of the stationary phase asymptotic of the integral on the LHS, and the formula means that the asymptotic gives exact answer. An explanation, according to Atiyah-Bott, is that $p=\omega+\hbar H$ is closed in the equivariant De Rham complex so that the LHS can be interpreted as the push forward of $\exp p$, while the RHS represents the same cohomology class via so called Borel localization theorem.

The localization theorem says (see [5] or [6]) that for a finite-dimensional $M$, pull-back from $M$ to the fixed point set $F$ induces an additive isomorphism between $H_{S^{1}}^{*}(M)$ and $H_{S^{1}}^{*}(F)=\oplus_{\alpha} H_{S^{1}}^{*}\left(F_{\alpha}\right)$ at least after localization to the field of rational functions $\mathbb{C}(\hbar)$. Additionally, it is easy to see that integration over the manifold $M$ transforms to integration over $F$ (which in symplectic situation is always a manifold) preceded by division by $E$ (which is always invertible in $H^{*}(F, \mathbb{C}(\hbar))$ ).

Notice that in this very famous theorem the algebraic-geometrical operation of 'localization' applies to the variable $\hbar$ which has cohomological nature. I think that an adequate understanding of mirror phenomenon requires systematic application of geometrical constructions to algebraic-topological objects: we will have to compute residues, integrate differential forms, solve differential equations and even do Morse theory in the spaces of (co)homological nature.

The following lemma gives another example from 'homological geometry' (some other, less trivial examples one can find in [8]).

Lemma-Example. Let $S^{1}$ act on $\mathbb{C}^{N}$ by diagonal matrices $\operatorname{diag}\left(e^{i \tau r_{1}}, \ldots, e^{i \tau r_{N}}\right)$. Then

$$
H_{S^{1}}^{*}\left(\mathbb{C} P^{N-1}\right)=\mathbb{C}[p, \hbar] /\left(\left(p-r_{1} \hbar\right) \ldots\left(p-r_{N} \hbar\right)\right) .
$$

The push forward operation is given by the formula

$$
f(p, \hbar) \mapsto \frac{1}{2 \pi i} \oint \frac{f(p, \hbar) d p}{\left(p-r_{1} \hbar\right) \ldots\left(p-r_{N} \hbar\right)} .
$$


This lemma presumes the following explicit description for $p$. Let $\omega$ be the standard symplectic Kahler form on $\mathbb{C} P^{N-1}$ obtained by the symplectic reduction of the standard form $\sim \sum d z_{j} \wedge d \bar{z}_{j}$ in $\mathbb{C}^{N}$ on the unit level of the Hamiltonian $\sum\left|z_{j}\right|^{2}$. Let $H$ be the Hamiltonian of the $S^{1}$-action obtained by such reduction from $\sum r_{j}\left|z_{j}\right|^{2}$. Let $\theta$ be the standard contact 1 -form on $E S^{1}=S^{\infty}$ (its differential equals the pull back of $\hbar$ on $\mathbb{C} P^{\infty}$ ). Then the 2-form $\Omega=\omega+d(H \theta)$ on $\mathbb{C} P^{N-1} \times E S^{1}$ is closed and projectable to the quotient by $S^{1}$ (see for instance M.Audin [5]). Therefore it determines an equivariant cohomology class of $\mathbb{C} P^{N-1}$ which we denote $p$. Its restriction to fibres over $B S^{1}$ coincides with $[\omega] \in H^{2}\left(\mathbb{C} P^{N-1}\right)$. In the De Rham model $p$ is represented by $\omega+\hbar H$ where the hamiltonian $H$ of the circle action should be shifted to have critical values $r_{1}, \ldots, r_{N}$.

Proof. The equivariant cohomology algebra in question coincides with the equivariant cohomology of $\mathbb{C}^{N}-0$ with respect to the action of $S^{1} \times T^{1}$ where $T^{1}$ is the scalar action, and the answer is well known in the theory of toric varieties (see [5]). Independently on what an intelligent proof of the push forward formula could be, it follows from Duistermaat-Heckmann formula which expresses the push forward as the sum of residues of the 1-form above.

\section{$2 \quad$ Floer homology and $\mathcal{D}$-modules}

We intend to explain here a rather general mechanism that relates algebraic geometry of rational curves in Kahler manifolds with linear PDE.

Let a compact complex manifold $M$ be provided with a holomorphic action of the complex circle $\mathbb{C}-0$. Whenever we are given an invariant Kahler form on $M$ the action of the real circle $S^{1} \subset(\mathbb{C}-0)$ turns into a locallyhamiltonian action with the Hamilton function $H$ (while the 'imaginary' part $\mathbb{R}_{+} \subset(\mathbb{C}-0)$ of the complex circle determines the action of the gradient flow of $H$ with respect to the real part of the Kahler metric).

Imagine that $H$ is multiple-valued indeed and becomes well defined only on a covering $\tilde{M} \rightarrow M$. Consider $\tilde{M}$ as a symplectic manifold with the symplectic form $\omega$ and the $H$-hamiltonian $S^{1}$-action lifted from $M$ and introduce the notations: $q$ - for a covering transformation $\tilde{M} \rightarrow \tilde{M}$, and $p$ - for the equivariant closed 2 -form $\omega+\hbar H$ on $\tilde{M}$ (normalized in such a way that 
$q^{*}(H)=H-1$ and therefore $\left.q^{*}(p)=p+\hbar\left(q^{*} H-H\right)=p-\hbar\right)$.

Consider $p$ and $q$ as operators of wedge-product by $p$ and of pull-back by $q$ acting on the equivariant De Rham complex or on its cohomology. Then we find the following remarkable commutation relation:

$$
p q-q p=\hbar q
$$

This commutation relation has two famous representations:

- coordinate, $p=\hbar \frac{d}{d t}, q=e^{t} \times$, acting on functions of exp $t$, and

- impulse, acting on functions of $p$ as the shift $q: p \mapsto p-\hbar$ and as multiplication by $p$ respectively.

In other words, the $S^{1}$-equivariant cohomology of $\tilde{M}$ should carry the structure of a (holonomic) module over the 'Heisenberg' algebra $\mathcal{D}$ of differential operators (or its finite-difference Fourier partner) on a circle, more generally - torus. As far as I know this is one of very few occurrences of non-commutative objects in algebraic topology.

'Unfortunately' the domain of this construction does not contain finitedimensional Kahler manifolds: as it follows from a simple argument involving Lefschetz' isomorphism, a non-hamiltonian symplectic action on such a manifold does not have fixed points.

We intend to apply this construction - informally - to the case where $M$ is the space $L X=\operatorname{Maps}_{0}\left(S^{1}, X\right)$ of free contractable loops in a compact Kahler manifold $X$. The loop space inherits all the structures (complex, Riemannian, symplectic, Kahler) that $X$ has. For instance, if $\omega$ is a symplectic form on $X$ and $v, w$ are two vector fields on $X$ along a loop $\gamma$ then the formula

$$
\oint \omega(v(t), w(t)) d t
$$

determines a symplectic form on $L X$. Besides this, $L X$ has a circle action induced by translations on the source $S^{1}$. This action preserves the symplectic form on $L X$ (since $d t$ is translation-invariant). It is easy to see that the corresponding Hamilton function $H$ is the action functional : to a contractable loop $\gamma$, it assigns the symplectic area of a disk $D$ contracting the loop,

$$
H(\gamma)=" \oint_{\gamma} x d y "=\iint_{D} \omega .
$$


Since another disk with the same boundary may have different area, the action functional is (usually) multiple-valued with periods determined by symplectic periods of $\omega$ on $\pi_{2}(X)$.

The localization theorem for equivariant cohomology does not apply literally to the infinite-dimensional loop space. However the cohomology theory that we intend to use for loop spaces is also not the usual one but rather Floer's or semi-infinite. This means that the cochain complex should be constructed from Morse cells of the action functional $H$ (they always have infinite dimension and infinite codimension). The remarkable observation (exploited by Floer [8]) that the gradient flow of the action functional consists in analytic continuation of loops to holomorphic cylinders relates Floer homology theory with geometry of holomorphic curves in $X$.

In this paper we need $S^{1}$-equivariant Floer cohomology of the universal covering $\tilde{L X}$. Such Floer cohomology is not easy to define rigorously (cf. $[7],[8],[9])$ but very easy to compute. The equivariant Morse-Smale-BottNovikov-Floer complex, by definition, equals the cohomology of the critical set of the action functional. The critical points (= constant loops) form a copy of the manifold $X$ duplicated at each 'floor' of the covering $\tilde{L X} \rightarrow L X$. The boundary operator in the complex should be taken trivial (due to a geometrical argument of Morse-theoretic nature that proves the corresponding finite-dimensional theorem, see [10] for more detail). Therefore the Floer cohomology in question is, as an additive object,

$$
F H_{S^{1}}^{*}(\tilde{L X})=H^{*}\left(X, \mathbb{C}\left[q, q^{-1}\right](\hbar)\right) .
$$

Here $\mathbb{C}\left[q^{ \pm 1}\right]$ stands for the group algebra of the lattice $\pi_{2}(X)$ which we identify with $\mathbb{Z}^{k}$ by choice of a basis so that $q=\left(q_{1}, \ldots, q_{k}\right)$. (In many cases one needs some completion of the Laurent polynomial algebra - exactly as in Morse-Novikov theory.)

According to the original Floer's viewpoint, Floer cohomology does not have a natural multiplicative structure, but bears a module structure over the usual cohomology (one can intersect semi-infinite cycles with cycles of finite codimension). Assuming for simplicity that $X$ is simply connected we can choose a basis of symplectic forms in $H^{2}(X)$ dual to the basis chosen in the lattice $\pi_{2}(X)=H_{2}(X)$, construct corresponding $S^{1}$-invariant symplectic forms $\omega_{1}, \ldots, \omega_{k}$ and action functionals $H_{1}, \ldots, H_{k}$ on the loop space $\tilde{L X}$ and define equivariant 2 -forms $p_{i}=\omega_{i}+\hbar H_{i}, i=1, \ldots, k$. 
We come to the conclusion that equivariant Floer cohomology bears the structure of a module over the algebra $\mathcal{D}$ of operators $p_{1}, \ldots, p_{k}, q_{1}^{ \pm 1}, \ldots, q_{k}^{ \pm 1}$ satisfying the relations

$$
p_{i} p_{j}=p_{j} p_{i}, q_{i} q_{j}=q_{j} q_{i}, p_{i} q_{j}-q_{j} p_{i}=\delta_{i j} \hbar q_{i}, i, j=1, \ldots, k .
$$

This $\mathcal{D}$-module structure in equivariant Floer cohomology explains the role that differential equations play in algebraic geometry of rational curves.

1. Since $\mathcal{M}=F H_{S^{1}}^{*}(\tilde{L X})$ (considered as a space of functions of $q$ with values in $\left.H^{*}(X)\right)$ is a free $\mathbb{C}\left[q^{ \pm 1}\right]$-module of finite rank $\left(=\operatorname{dim} H^{*}(X)\right)$, the $D$-module in question has a good chance to be holonomic. According to general theory (thanks to M.Kapranov who taught me this construction), such a $\mathcal{D}$-module determines a locally constant sheaf

$$
\operatorname{Hom}_{\mathcal{D}}(\mathcal{M}, \mathcal{O}) .
$$

Here $\mathcal{D}$ is the algebra of differential operators on the torus with coordinates $q_{1}, \ldots, q_{k}$, and $\mathcal{O}$ is the sheaf of holomorphic functions on the torus. In this way we encode the $\mathcal{D}$-module structure in equivariant Floer cohomology by means of a flat connection in the bundle over the torus with the fibre $H^{*}(X)$.

2. While the $D$-module structure and the locally flat connection described above have invariant meaning, the differential equations arise in a noninvariant way when one considers resolutions $\mathcal{M} \leftarrow \mathcal{D}^{N} \leftarrow \ldots$ and thus chooses generators. However in our situation there is a canonical choice of a vector space generating $\mathcal{M}$. Consider a cycle $\Gamma$ in $X$ and generate a semi-infinite cycle in $\tilde{L X}$ as the union of all gradient trajectories of the action functional outgoing $\Gamma$ considered as a critical set on the 'zero' floor of the covering $L \tilde{X} \rightarrow L X$. The semi-infinite cycle consists of all those algebraic loops which are boundary values of holomorphic maps $\varphi:\{|z| \leq 1\} \rightarrow X$ of the unit disk, with $\varphi(0) \in \Gamma$. It is independent on the choice of the action functional and invariant with respect to the circle action on $\tilde{L X}$. Therefore it represents an equivariant Floer class in $\mathcal{M}$.

In this way we embed $H^{*}(X)$ to $\mathcal{M}$ as a subspace of 'geometrical cycles' generating $\mathcal{M}$ over $\mathbb{C}\left[q^{ \pm 1}\right]$. In coordinates, if $c_{1}, . .,, c_{N}$ is a basis in 
$H^{*}(X)$, a local homomorphism $\mathcal{M} \rightarrow \mathcal{O}$ is given by $N$ local functions $f_{1}, \ldots, f_{N}$ of $q$. Since

$$
p_{i} c_{\alpha}=\sum_{\beta} a_{i \alpha}^{\beta} c_{\beta}
$$

where $a$ 's are some functions of $q$ (and may be $\hbar$ ), and $p_{i}$ act on $f_{\alpha} \in \mathcal{O}$ as $\hbar q_{i} \partial / \partial q_{i}$, we obtain the differential equations

$$
\hbar q_{i} \frac{\partial}{\partial q_{i}} f_{\alpha}=\sum_{\beta} a_{i \alpha}^{\beta} f_{\beta}, i=1, \ldots, k
$$

expressing the fact that homomorphisms $\mathcal{M} \rightarrow \mathcal{O}$ are horizontal sections of our locally flat connection.

It is that system of linear PDE which one usually considers in connection with quantum cohomology theory (see [9], [10] for more detail and references).

3. More generally, one can pick an element $c \in \mathcal{M}$ and generate a $\mathcal{D}$ submodule $\mathcal{D} c$ which is therefore identified with the quotient $\mathcal{D} / I_{c}$ by the left ideal annihilating $c$. Generators of the ideal are the 'higher order linear differential equations satisfied by $c$ '.

This construction is especially convenient if the cohomology algebra $H^{*}(X)$ is multiplicatively generated by 2-forms (as in the case of toric or flag manifolds). In such a case one can choose $c$ to be 'the fundamental semi-infinite cycle' i. e. apply the above construction of equivariant Floer cycles to the fundamental cycle $\Gamma$ in $X$. Such $c$ consists of boundary values of all holomorphic disks in $X$ and, under the assumption made, generates $\mathcal{M}$ over $\mathcal{D}$ (without the assumption it generates however a submodule of principal importance in connection with rational curves).

4. Passing to the quasi-classical limit $\hbar \rightarrow 0$ from $\mathcal{D}$ to the algebra of commuting variables $p, q$ with the Poisson bracket

$$
\left\{p_{i}, q_{j}\right\}=\delta_{i, j} q_{i}
$$

one obtains from the left ideal $I_{c}$ a commutative Poisson ideal $I$ in the Poisson algebra $\mathbb{C}\left[p, q^{ \pm 1}\right]$ of function on the cotangent bundle of the 
torus. The ideal $I$ can be interpreted as an ideal of equations of some Lagrangian subvariety $L$ in the cotangent bundle - the characteristic variety of the $\mathcal{D}$-module $\mathcal{D} / I_{c}$.

The algebra $\mathbb{C}[p, q] / I$ is nothing but the quantum cohomology algebra of $X$ (or a subalgebra in it generated by $H^{2}(X)$, see [9], [10] for various constructions of Witten's quantum cohomology).

In particular this means that such quantum cohomology algebras are algebras $\mathbb{C}[L]$ of functions on Lagrangian varieties (cf. [10]) or, in other words, that the Poisson bracket $F, G$ of two relations between $p$ and $q$ in a 'Floer-Witten cohomology algebra' is a relation again.

Of course, constructions described above, are far from being rigorous mathematical definitions. However we will see in examples how they work, what are the differential equations, and even will be able to write down complete solutions of these differential equations in cohomological terms.

\section{Example: Projective spaces}

The complex projective space $\mathbb{C} P^{n-1}$ can be considered either as the quotient of $\mathbb{C}^{n}-0$ by the diagonal action of the complex 'torus' $T_{\mathbb{C}}=\mathbb{C}-0$ or as a symplectic quotient of $\mathbb{C}^{n} / / T$ by its real subtorus $T \subset T_{\mathbb{C}}$.

We are going to study the 'semi-infinite topology' of the space

$$
M_{\infty}=(\text { non-zero } n \text {-tuples of polynomials in } z) / T_{\mathbb{C}} \text {. }
$$

It has finite-dimensional approximations by the analogous spaces $M_{d}$ with the degree of polynomials bounded by $d$. On one hand, $M_{d}$ is a compactification of the space of degree $d$ parametrized rational curves in $\mathbb{C} P^{n-1}$. On the other hand, the limit $M_{\infty}$ of these spaces can be treated as Floer's fundamental cycle in the projectivized space of $n$-tuples of Laurent polynomials in $z$. The latter, let us denote it $M$, is a good substitute for the universal covering of the space of algebraic loops in $\mathbb{C} P^{n-1}$. The covering transformation group $\mathbb{Z}$ acts on $M$ by simultaneous multiplication of the $n$ polynomials by (a power of) $z$.

The other circle, $S^{1}$, acts on $M$ by rotation of loops $z \mapsto e^{2 \pi i \phi} z$.

Now we are going to realize our 'equivariant Floer homology' program, at first - using very simple formal computations with divergent infinite 
products, and then reproduce the same results using only well-defined mathematical objects.

1. According to our Lemma-Example, $S^{1}$-equivariant cohomology of $M$ (computed as a limit of such cohomology for finite-dimensional approximations of $M$ using Laurent polynomials of bounded degrees) is

$$
\mathbb{C}[p, \hbar] / \Pi_{m=-\infty}^{\infty}(p-m \hbar)^{n} .
$$

(whatever it means!)

The fundamental semi-infinite cycle $\left[M_{\infty}\right] \subset M$ is Poincare-dual to the class

$$
\Delta=\Pi_{m=1}^{\infty}(p+m \hbar)^{n} .
$$

Since $q(p)=p-\hbar$, we find that $\Delta$ satisfies the finite-difference equation $q \Delta=p^{n} \Delta$. Therefore the left ideal $I_{\Delta}$ in the algebra $\mathcal{D}$ is generated by $p^{n}-q$.

In the 'coordinate' representation of differential operators it transforms to the differential equation

$$
\left(\hbar \frac{d}{d t}\right)^{n} f(t)=e^{t} f(t)
$$

In the quasi-classical limit this 'PDE' generates the Lagranginan variety $L=\left\{(p, q) \mid p^{n}=q\right\}$ while the symplectic structure is $q^{-1} d p \wedge d q$.

The algebra $\mathbb{C}[L]=\mathbb{C}\left[p, q^{ \pm 1}\right] /\left(p^{n}-q\right)$ is the quantum cohomology algebra of the complex projective space, and the differential equation in question is the one that the quantum cohomology theory associates to $\mathbb{C} P^{n-1}$.

2. Consider the $\mathcal{D}$-module $\mathcal{M}=\mathcal{D} / I_{\Delta}$ and compute the space of solutions $\operatorname{Hom}_{\mathcal{D}}(\mathcal{M}, \mathcal{O})$ of our differential equation. It seems to be a general rule that such a space has an explicit description in cohomological terms.

Consider the Borel localization isomorphism of equivariant cohomology of $M$ with that of the fixed point manifold. The latter consists of copies of $\mathbb{C} P^{n-1}$ numbered by the elements $k \in \mathbb{Z}$ of the covering transformation lattice (a Laurent $n$-tuple represents a fixed point if and only if it is $\left.\left(a_{1} z^{k}: \ldots: a_{n} z^{k}\right)\right)$. 
Denote $C$ an equivariant cohomology class which has $\mathbb{Z}$-invariant localizations (i. e. restricts to the same cohomology class in each copy of $\left.\mathbb{C} P^{n-1}\right)$. Then the equivariant classes $\Gamma_{C}=e^{p t / \hbar} C$ satisfy

$$
p \Gamma_{C}=\hbar \frac{d}{d t} \Gamma_{C}, q^{*} \Gamma_{C}=e^{t} \Gamma_{C}
$$

and thus provide the Fourier transform from the impulse to the coordinate representation, in the following sense:

$$
\left\langle\Gamma_{C}, \cdot\right\rangle: \mathcal{M} \rightarrow \mathcal{O}
$$

is a homomorphism over $\mathcal{D}$. Here $\langle$,$\rangle means equivariant intersection$ index with values in $\mathbb{C}[[\hbar]]$, and $q^{*}$ — the operator adjoint to $q$.

Therefore $f_{C}(t)=\left\langle e^{p t / \hbar} C, \Delta\right\rangle$ are solutions to our differential equation.

3. Explicitly, according to the second part of our Lemma-Example,

$$
\begin{gathered}
f_{C}(t)=\frac{1}{2 \pi i} \oint \frac{e^{p t / \hbar} C d p}{p^{n}(p-\hbar)^{n}(p-2 \hbar)^{n} \ldots} \\
=\hbar^{1-n} \sum_{d=0}^{\infty} \frac{e^{d t}}{\hbar^{n d}} \operatorname{Res}_{0} \frac{e^{P t} C d P}{P^{n}[(P+1)(P+2) \ldots(P+d)]^{n}} \frac{1}{\Pi_{m=1}^{\infty}(\hbar(P-m))^{n}} .
\end{gathered}
$$

Here $P=(p-d \hbar) / \hbar$ can be considered as a nilpotent variable, $P^{n}=0$, and $C$ - as a polynomial in $P$. Notice that $\mathbb{C}[P] /\left(P^{n}\right)$ is the cohomology algebra of $\mathbb{C} P^{n-1}$, and $\langle a, b\rangle=\operatorname{Res}_{0} a b d P / P^{n}$ is the intersection form in the cohomology. The function $f_{C}(t)$ is still not well-defined because of the divergent infinite product factor on the right. It is however the same in each summand and independent of $t(!)$. We redefine $f$ simply by dropping this factor and thus come to the following remarkable formula for general solution of the differential equation $\hbar^{n} f^{(n)}=e^{t} f$ :

$$
f_{C}(t)=\hbar^{1-n}\left\langle\sum_{d=0}^{\infty} \frac{e^{d t}}{\hbar^{n d}} \frac{e^{P t}}{[(P+1)(P+2) \ldots(P+d)]^{n}}, C(P)\right\rangle .
$$

4. The $b r a$ - part of the above formula gives an expansion in $q=\exp (t)$ and $\log (q)$ of the vector-function $\vec{f}$ with values in cohomology of $\mathbb{C} P^{n-1}$ 
whose components are solutions of our ODE. It can be rewritten in the form

$$
\vec{f}(t, \hbar)=\exp (P \log q)\left[f_{0}(Q)+f_{1}(Q) P+\ldots+f_{n-1}(Q) P^{n-1}\right]
$$

where $Q=q / \hbar$. It is clear now that the solutions have unipotent monodromy around $q=0$. The first component is monodromy-invariant and is given by the following 'hyper-geometric' series

$$
f_{0}=\sum_{0}^{\infty} \frac{Q^{d}}{(d !)^{k}} \text {. }
$$

The next component has the form $t f_{0}(Q)+f_{1}(Q)$ with $f_{1}(0)=0$, and so on.

Theorem. Let $A$ denote the Hamiltonian of the $S^{1}$-action on the projective space $M_{d}$ of degree-d rational maps to $\mathbb{C} P^{n-1}, \omega$ - the standard Fubbini symplectic form on $M_{d}$. Then

$$
\sum_{d=0}^{\infty} e^{d \tau} \int_{M_{d}} e^{(t-\tau)(A+\omega / \hbar)}=\hbar^{1-n}\langle\vec{f}(t, \hbar), \Omega \vec{f}(\tau,-\hbar)\rangle
$$

where $\Omega$ is the automorphism $\Omega(P)=-P$ of $\mathbb{C}[P] /\left(P^{n}\right)$.

Proof: One derives this from the Duistermaat-Heckmann formula by means of straightforward computations similar to those which we have just completed, but without any 'dirty tricks'.

The automorphism $\Omega$ can be replaced by the change of notations $P:=\hbar P$.

This theorem assigns precise geometrical meaning to the differential equation we are studying and to its solutions: the RHS is a function of two variables satisfying essentially the same ODE in each of them, and thus picking various values for $\tau$ we will obtain a basis of solutions in $t$.

On the other hand, the theorem explains also our original 'naive' computation. Actually we were computing some Feynman integral, $\int \exp (t(A+$ $\omega / \hbar)$ ), over $M_{\infty}$ or a finite codimension subspace therein. We have found that the integral formally satisfies our differential equation but probably diverges. The LHS in the theorem is therefore a 'regularization' of the Feynman integral in terms of approximations of $M_{\infty}$ by $M_{d}$; it involves shifting the symplectic class $t \omega$ by $\tau \omega$ and replacing the divergent sequence by its generating series in $\exp \tau$. 


\section{Hypersurfaces in $\mathbb{C} P^{n-1}$}

Let us try now to study semi-infinite topology of spaces of rational curves on hypersurfaces in $\mathbb{C} P^{n-1}$ using equations of the hypersurfaces.

Let $F\left(x_{1}: \ldots: x_{n}\right)=0$ be a homogeneous equation of degree $l$ of such a hypersurface $X$. In the projective space $M_{d}$ of $n$-tuples $\left(x_{1}(z), \ldots, x_{n}(z)\right)$ of polynomials of degree $\leq d$, the condition $F(x(z))=0$ for all $z$ describes a subvariety invariant with respect to the Möbius group. Let us find the equivariant cohomology class that such a subvariety would represent if its equations were non-singular.

The degree of the polynomial $F(x(z))=A_{0}+A_{1} z+\ldots+A_{l d} z^{l d}$ does not exceed $l d$ and its coefficients $\left(A_{0}, \ldots, A_{l d}\right)$ (considered as polynomials of degree $l$ of the $n(d+1)$ coefficients of polynomials $\left.x_{j}(z)\right)$ form a section of an $(l d+1)$-dimensional vector bundle over $M_{d}$. It is easy to see that this vector bundle is the tensor product of the $(-l)$-th power of the Hopf line bundle over $M_{d}$ with the trivial vector bundle whose fiber is the space $V$ of polynomials of degree $l d$ in one variable $z$. The vector bundle is Möbius-equivariant i. e. the base and the space bear compatible actions of the Möbius group, and our section is Möbius-invariant.

If the section was transverse to the zero section, its zero locus would represent the equivariant Euler class of the vector bundle, and this is the class that we are going to compute, replacing as usually the Möbius group with its maximal torus $S^{1}$. By the equivariant Euler class of a $G$-equivariant bundle $W \rightarrow Y$ we mean of course the usual Euler class of the bundle $W_{G} \rightarrow Y_{G}$.

One can compute the $S^{1}$-equivariant Euler class by simply looking at the weights of the circle action. With our standard notations $(p, \hbar)$ for the generators of the equivariant cohomology of $M_{d}$ the Euler class is

$$
E_{d, l}=l p(l p-\hbar)(l p-2 \hbar) \ldots(l p-l d \hbar) .
$$

Now passing to the limit $d \rightarrow \infty$ we perform the same formal operations as in the previous section, but with the $\mathcal{D}$-module generated by

$$
\Delta_{l}=\Pi_{m=0}^{\infty}(l p-m \hbar)
$$

in the equivariant cohomology of $M_{\infty}$. A motivation for this is the naive idea that the class $\Delta_{l}$ should represent the Floer fundamental cycle in $\tilde{L X}$ for our projective hypersurface $X$. 
1. The expression

$$
\Delta \cdot \Delta_{l}=\Pi_{m=1}^{\infty}(p+m \hbar)^{n} \Pi_{m=0}^{\infty}(l p-m \hbar)
$$

satisfies the finite-difference equation

$$
p^{n-1} \Delta \cdot \Delta_{l}=q\left[l(l p+\hbar)(l p+2 \hbar) \ldots(l p+(l-1) \hbar) \Delta \cdot \Delta_{l}\right] .
$$

In coordinate representation this relation becomes the differential equation

$$
\hbar^{n-l}\left(\frac{d}{d t}\right)^{n-1} f=e^{t} \cdot l\left(l \frac{d}{d t}+1\right) \ldots\left(\frac{d}{d t}+l-1\right) f .
$$

Notice that for $n=l=5$ it is exactly the ODE from Introduction!

2. Solutions of this equation can be found in the form

$$
\left\langle e^{p t / \hbar} C, \Delta \cdot \Delta_{l}\right\rangle=\frac{1}{2 \pi i} \oint \frac{e^{p t / \hbar} l p(l p-\hbar)(l p-2 \hbar) \ldots C d p}{p^{n}(p-\hbar)^{n}(p-2 \hbar)^{n} \ldots} .
$$

Notice that the nilpotent variable $P=(p / \hbar-d)$ satisfies $P^{n-1}=0$ since each residue has the form

$$
\text { (invertible function) } C \frac{l P d P}{P^{n}} \text {. }
$$

Rewriting the sum of residue as an intersection index we find ourselves in the algebra $\mathbb{C}[P] /\left(P^{n-1}\right)$ with the intersection pairing $\left\langle P^{a}, P^{b}\right\rangle=l$ for $a+b=n-2$ and $=0$ otherwise. This is exactly the image of $H^{*}\left(\mathbb{C} P^{n-1}\right)$ in the cohomology algebra $H^{*}(X)$ of the hypersurface with the intersection index of cycles in $X$.

3. In these notations the solutions are

$$
f_{C}=\hbar^{2-n}\left\langle e^{P t} \sum_{d=0}^{\infty}\left(\frac{e^{t}}{\hbar^{n-l}}\right)^{d} \frac{(l P+1) \ldots(l P+l d)}{[(P+1) \ldots(P+d)]^{n}}, C(P)\right\rangle .
$$

Notice that this formula gives $n-1$ independent solutions which means that for $l>n$ we should rather think of our $(l-1)$-st order differential equation as of 'an $(n-1)$-st order pseudo-differential one'. (By the way, this indicates how quantum cohomology theory should be affected by the negative 1 -st Chern class of $X$, cf. [9]). 
4. The solutions have unipotent monodromy around $q=0$, and the invariant solution is given by the following hypergeometric series of $Q=e^{t} / \hbar^{n-l}$ :

$$
f_{0}(Q)=\sum_{d=0}^{\infty} \frac{(l d) !}{(d !)^{n}} Q^{d}
$$

One can also interpret $\hbar^{2-n}\langle\vec{f}(t, \hbar), \vec{f}(t,-\hbar)\rangle$ as the generating function

$$
\sum_{d=0}^{\infty} e^{d \tau} \int_{M_{d}} E_{d, l} e^{(t-\tau)(A+\omega / \hbar)}
$$

for the sequence of integrals in $M_{d}$ over equivariant Euler cycles.

One more rigorous (but a bit ugly) interpretation of $\vec{f}$ : computing the integrals $\int e^{p t / \hbar} E_{d, l} C$ over $M_{d}$ one should confine only contributions of global maxima of $A$ and take the sum over all $d$.

Our computation can be easily generalized to complete intersections in toric manifolds — we will write out the details elsewhere.

\section{Quintics in $\mathbb{C} P^{4}$}

Consider now the case when $X$ is a quintic hypersurface in $\mathbb{C} P^{4}$. It is known that on a generic quintic rational curves are situated in a discrete manner indeed, and we can pretend that their numbers $n_{d}$ are known, in order to compute the $\mathcal{D}$-module $\mathcal{M}$ for $X$ directly.

The complex dimension, predicted by the Riemann-Roch formula, of the space of algebraic loops in $X$ equals 3 , and the loops themselves are represented by various holomorphic parametrizations of the rational curves in $X$. Therefore the space $\tilde{L} \tilde{X}_{\text {alg }}$ should have many irreducible components attached to each other in a rather complicated manner. Instead of computing equivariant cohomology of this complicated space we intend to compute the 'Feynman path integrals' $\int \exp (p T / \hbar)$ over Floer cycles as the sum over the 3-dimensional components and then find the differential equation to which these integrals satisfy as functions of $T$. 
The Floer fundamental cycle, by 'definition', consists of all gradient trajectories of the action functional outgoing the critical set $X$ on the zero floor of the covering $\tilde{L X} \rightarrow L X$. It includes (1) constant loops, (2) Möbius reparametrizations of rational curves in $X,(3)$ multiple covers of such curves.

1. Constant maps

Their contribution is given by the integral

$$
\int_{X} e^{p T / \hbar} C=\hbar^{-3} \int_{X} e^{P T} C(P)=\hbar^{-3}\left\langle e^{P T}, C(P)\right\rangle
$$

where $P^{4}=0$ and $\left\langle P^{3}, 1\right\rangle=5$.

2. Embeddings $\mathbb{C} P^{1} \subset X$ of degree d

Consider a rational curve of degree $d$ in $X$. The space of degree-1 maps from $\mathbb{C} P^{1}$ to this curve compactifies into the projective space $\mathbb{C} P^{3}$ of pairs of linear polynomials. This projective space can be considered as a compactified irreducible component of the space $\tilde{L X}_{a l g}$. The action of $S^{1}$ on $\mathbb{C} P^{3}$ has two copies of $\mathbb{C} P^{1}$ as the fixed point set. They correspond to constant loops on zero and $d$-th floors of the covering, namely to the constant loops with values in our rational curve in $X$.

Denote $r$ the generator in the equivariant cohomology algebra $\mathbb{C}[r, \hbar] /\left(r^{2}(r-\hbar)^{2}\right)$ of $\mathbb{C} P^{3}$. Since our rational curve has degree $d$ in $X$, $r=p d$ where $p$ stands for our usual equivariant class $\omega+A \hbar$ on $\tilde{L X}$. Contribution of this $\mathbb{C} P^{3}$ into the path integral equals

$$
\begin{gathered}
\int e^{p T / \hbar} C=\frac{1}{2 \pi i} \oint \frac{e^{p T / \hbar} C d r}{r^{2}(r-\hbar)^{2}}=d^{3} \hbar^{-3} \frac{1}{2 \pi i} \oint \frac{e^{\rho T} C d \rho}{\rho^{2}(\rho-d)^{2}} \\
=d^{3} \hbar^{-3}\left\langle e^{P T}\left[\frac{1}{(P-d)^{2}}+\frac{e^{d T}}{(P+d)^{2}}\right], C(P)\right\rangle
\end{gathered}
$$

where $P^{2}=0$ and $\langle P, 1\rangle=1$.

3. Multiple covers

The space of rational maps to $\mathbb{C} P^{1}$ of degree $m$ compactifies into $\mathbb{C} P^{2 m+1}$. It has 'wrong' dimension which means that such maps considered as algebraic loops represent non-transverse intersection points 
of two 'non-algebraic' Morse-Floer cycles — an 'up-going' cycle and a 'down-going' one. One takes care of this non-transversality in the following way.

The two cycles consist of loops which extend holomorphicly to the interior and exterior of the unit disk respectively. Non-transversality means that the sum of tangent spaces has positive codimension, and the quotient by this sum forms a conormal bundle over the actual intersection set. In order to compute contributions of this intersection set into intersection indices or integrals one should perturb the cycles to a general position. However, since the cycles are already 'transverse' in the most of directions (except for the conormal ones), instead of perturbing the cycles one can replace the fundamental cycle of the actual intersection by the Euler cycle of the conormal bundle.

Consider a generic point $\phi \in \mathbb{C} P^{2 m+1}$ in the actual intersection set. The intersection of tangent spaces to our cycles at $\phi$ is the space $H^{0}\left(\mathbb{C} P^{1}, \phi^{*} T X\right)$ of holomorphic sections of the tangent bundle to $X$ induced to $\mathbb{C} P^{1}$ by means of the map $\phi: \mathbb{C} P^{1} \rightarrow X$. Discreteness of all rational curves on generic quintics actually means that the normal bundle to such a curve in $X$ is $\mathcal{O}(-1) \oplus \mathcal{O}(-1)$. Therefore for a cover of multiplicity $m$ the induced tangent bundle

$$
\phi^{*} T X \simeq \mathcal{O}(2 m) \oplus \mathcal{O}(-m) \oplus \mathcal{O}(-m)
$$

(where the 1-st summand represents the pull-back of the tangent bundle to the curve in $X)$. In particular we observe that $H^{0}\left(\mathbb{C} P^{1}, \phi^{*} T X\right)=$ $T_{\phi} \mathbb{C} P^{2 m+1}$ and thus we are in a position to apply the 'conormal bundle trick' described above.

The conormal space is naturally identified with

$$
H^{1}\left(\mathbb{C} P^{1}, \phi^{*} T X\right) \simeq H^{0}\left(\mathbb{C} P^{1}, \mathcal{O}(-2 m-2) \oplus \mathcal{O}(m-2) \oplus \mathcal{O}(m-2)\right) .
$$

This space is the direct sum of two copies of the space $V$ of polynomials of degree $m-2$ in one variable $z$ and its dimension equals $2 m-2$ (it agrees with the Riemann-Roch formula, $(2 m+1)-(2 m-2)=3)$.

The computation we are performing has been actually done in detail by Aspinwall-Morrison in [11]. They showed that the conormal spaces 
really form a vector bundle over the compactified space of multiple covers i. e. over $\mathbb{C} P^{2 m+1}$, and computed the bundle. It is the direct sum of two copies of $\mathcal{O}_{\mathbb{C} P^{2 m+1}}(-1) \otimes V^{*}$ where $V$ is the trivial bundle with the polynomial space (denoted $V$ in the previous paragraph) as the fiber. This description takes in account the natural Möbius group action on the conormal bundle. Looking at the weights of this action, we can complete our computation: the $S^{1}$-equivariant Euler class of the conormal bundle in the equivariant cohomology algebra of $\mathbb{C} P^{2 m+1}$ equals

$$
(r-\hbar)^{2}(r-2 \hbar)^{2} \ldots(r-(m-1) \hbar)^{2} \in \mathbb{C}[r, \hbar] /\left(r^{2}(r-\hbar)^{2} \ldots(r-m \hbar)^{2}\right) .
$$

Therefore this component of the Floer fundamental cycle contributes to the integral as

$$
\begin{gathered}
\int e^{p T / \hbar} C=\frac{1}{2 \pi i} \oint \frac{e^{p T / \hbar} C d r}{r^{2}(r-m \hbar)^{2}}=d^{3} \hbar^{-3} \frac{1}{2 \pi i} \oint \frac{e^{\rho T} C d \rho}{\rho^{2}(\rho-d m)^{2}} \\
=d^{3} \hbar^{-3}\left\langle e^{P T}\left[\frac{1}{(P-d m)^{2}}+\frac{e^{d m T}}{(P+d m)^{2}}\right], C(P)\right\rangle
\end{gathered}
$$

where $\rho=p / \hbar, P^{2}=0$ and $\langle P, 1\rangle=1$.

Of course this formula for $m=1$ coincides with the previous one.

Now we should take the sum of these contributions over all rational curves in $X$.

First of all let us find the differential equation to which the sum satisfies. If we differentiate two times in $\hbar d / d T$ then all the terms disappear, except for

$$
d^{3} \hbar^{-1}\left\langle e^{P T} \cdot e^{d m T}, C(P)\right\rangle
$$

(where $P^{2}=0,\langle P, 1\rangle=1$ ) and one more term, from Constant maps, which can be written as $5\left\langle e^{P T}, C(P)\right\rangle$ where again $P^{2}=0$ and $\langle P, 1\rangle=1$. The total sum equals

$$
\hbar^{-1}\left\langle e^{P T}\left[5+\sum_{d=1}^{\infty} n_{d} d^{3} \sum_{m=1}^{\infty} e^{d m T}\right], C(P)\right\rangle .
$$


Therefore if we divide the sum by

$$
K\left(e^{T}\right)=5+\sum_{d=1}^{\infty} \frac{n_{d} d^{3} e^{d T}}{1-e^{d T}}
$$

we obtain $\hbar^{-1}\left\langle e^{P T}, C(P)\right\rangle$ which is annihilated by the 2-nd derivative in $T$. Thus our 'path integral' satisfies the equation

$$
\left(\frac{F^{\prime \prime}}{K}\right)^{\prime \prime}=0 \quad \text { where } \quad,=\hbar \frac{d}{d T} .
$$

On the other hand, the sum of all componentwise integrals actually diverges because of some infinite sums (proportional to $\sum n_{d} d^{3}=\infty$ ) in the constant and linear terms in $T$. In order to extract from our formulas the general solution to our differential equation, we should - exactly in the same way as we actually did in the previous section - in each Duistermaat-Heckman integral confine only its localization near the maximum of the Hamiltonian i. e. throw away all left residues and keep right ones. The resulting sum can be written in the uniform notations $P^{4}=0,\left\langle P^{3}, 1\right\rangle=5$, that is in terms of the even part of the cohomology algebra of $X$, as

$$
\begin{gathered}
\vec{F}(T)=e^{P T}\left(1+\frac{1}{5} \sum_{d=1}^{\infty} n_{d} d^{3} \sum_{m=1}^{\infty} \frac{P^{2} e^{d m T}}{(P+d m)^{2}}\right) \\
F_{C}(T)=\hbar^{-3}\langle\vec{F}(T), C(P)\rangle .
\end{gathered}
$$

Notice that the first two components of $\vec{F}$ equal 1 and $T$.

\section{The 'Yukawa coupling'}

Now we are ready to compare our two approaches to rational curves on quintics. The 'naive' approach based on embeddings of quintics into $\mathbb{C} P^{4}$ gave rise to the cohomology-valued vector-function

$$
\vec{F}(t)=e^{P t} \sum_{d=0}^{\infty} e^{d t} \frac{(5 P+1) \ldots(5 P+5 d)}{(P+1)^{5} \ldots(P+d)^{5}} .
$$

It should be compared with the 'mature' function $\vec{F}(T)$ found in the previous section.

The Mirror conjecture for quintics now says that 
The two curves, $\vec{F}$ and $\vec{f}$, considered as non-parametrized curves in the cohomology algebra $H^{\text {even }}(X)=\mathbb{C}[P] /\left(P^{4}\right)$ are proportional (with the function $f_{0}\left(e^{t}\right)=\sum(5 d) ! e^{d t} /(d !)^{5}$ as the proportionality coefficient),

or, in other words, that

Cones over the curves $\vec{F}$ and $\vec{f}$ concide.

We will show now how this form of the conjecture induces the 'strange' computational procedure described in Introduction.

First of all notice that the differential operators annihilating components of our curves are self-adjoint. This means that the differential equations are Euler-Lagrange equations of some variational principles and are therefore equivalent to some linear hamiltonian systems. In particular, the space of solutions bears a natural symplectic structure. For our equations, the space of solutions can be identified with the dual of the cohomology algebra $H^{\text {even }}(X)$ and one can find the symplectic structure to coincide with $\langle\cdot, \Omega \cdot\rangle$ where $\Omega\left(P^{i}\right)=(-P)^{i}$ is the diagonal operator from the end of Section 3 .

We claim that the cone in question is Lagrangian with respect to this symplectic form. Actually it is a general fact from the theory of linear variational ODE. A higher order linear ODE determines a curve in the dual projective space of the space of solutions: to a time moment $t$ the curve assigns the hyperplane of all solutions vanishing at this moment. If the equation is variational, then the space of solutions is symplectic and the curve is self-dual: its osculating hyperplane is skew-orthogonal to its application point (and the whole osculating flag is self-dual i. e. coincides with the flag of the skeworthogonal complements). In dimension 4 such self-duality means that the cone over the curve is Lagrangian.

Now let us figure out a geometrical meaning of the Yukawa coupling in the generality suitable for Calabi-Yau 3-folds. Given a Lagrangian submanifold $L$ in a linear symplectic space (a maximal family of algebraic Calabi-Yau 3-folds $Y$ always determines a Lagrangian manifold in $H^{3}(Y)$ ), one can define its 'symplectic curvature' which turns out to be a totally symmetric 3 -differential on $L$. Indeed, in a neighborhood of a point $x \in L, L$ can be identified with a section of the cotangent bundle $T^{*}\left(T_{x} L\right)$ of its tangent plane. Such a Lagrangian section is the graph of the differential $d \phi$ of some function 
$\phi$ on $L$ vanishing at $x$. In our situation $d_{x} \phi$ and $d_{x}^{2} \phi$ also vanish and thus $d_{x}^{3} \phi$ is a well-defined 3 -tensor on $T_{x} L$. One can see that it is independent on the choice of the cotangent bundle structure in the space.

If the Lagrangian submanifold is conical and therefore determines a Legendrian submanifold in the contact projective space, one can interpret the 3-differential as the 'contact curvature' - a totally symmetric 3-differential on the Legendrian submanifold, with values in the 2-nd tensor power of the Hopf bundle.

In any way, in our simple situation of the Lagrangian cone over a parametrized curve $\vec{f}(t)$ the 3 -differential restricted to the curve is

$$
Y(t)=\left\langle\overrightarrow{f^{\prime \prime \prime}}(t), \Omega \vec{f}(t)\right\rangle(d t)^{\otimes 3} .
$$

When the curve is replaced with a proportional curve $\psi \vec{f}$, the 3-differential transforms to $\psi^{2} Y$. If the curve is given by a variational differential equation, the symplectic curvature can be read directly from the quadratic Hamiltonian $H$ : it is essentially $H d t$ where the quadratic form $H$ should be restricted to the tangent plane of our Lagrangian submanifold. Therefore one can express the 3-differential through coefficients of the differential equation.

Since we are in a possession of solutions of our differential equations we can find the 'Yukawa coupling' $Y$ directly.

For the curve $\vec{f}$

$$
\begin{aligned}
Y(t)= & {\left[\left.\left(\frac{d}{d t}\right)^{3}\right|_{\tau=t} \sum_{d} e^{d \tau} \hbar^{3} \int_{M_{d}} e^{p(t-\tau) / \hbar} E_{d, 5}\right](d t)^{3} } \\
= & {\left[\sum_{d} e^{d t} \frac{1}{2 \pi i} \oint \frac{p^{3} E_{d, 5}(p, \hbar) d p}{p^{5} \ldots(p-d \hbar)^{5}}\right](d t)^{3} } \\
& =\left[\sum_{d} e^{d t} 5^{5 d+1}\right](d t)^{3}=\frac{5(d t)^{3}}{1-5^{5} e^{t}} .
\end{aligned}
$$

On the other hand, from the explicit formula for $\vec{F}$ we find

$$
\vec{F}^{\prime \prime \prime}=K\left(e^{T}\right) P^{3} / 5
$$


and therefore

$$
Y(T)=K\left(e^{T}\right)(d T)^{3}
$$

If our two curves are proportional indeed, then the 3-differentials will coincide after suitable transformations. The 'mature' curve has the form $1+P T+\ldots$ and the 'naive' one is $f_{0}\left(e^{t}\right)+P\left(t f_{0}\left(e^{t}\right)+f_{1}\left(e^{t}\right)\right)+\ldots$ where '...' mean terms with $P^{2}$ and $P^{3}$. Thus if we divide $\vec{f}$ by $f_{0}$ the curves will coincide up to parametrization. This 'renormalizes' $Y(t)$ to the form from Introduction. On the other hand it identifies $T$ with $t+f_{1}\left(e^{t}\right) / f_{0}\left(e^{t}\right)$ and thus shows how the 'mature' parameter $T$ can be found from solutions of the 'naive' equation.

Although the conjecture that the curves are proportional is a statement about two objects of the same nature, it still remains open. According to a remark of E.Witten in the paper [12] (where he studies models of CTFT also based on embeddings of Calabi-Yau hypersurfaces into projective spaces), one should expect the two curves to coincide after a "renormalization procedure' which changes the magnitude and parametrization only: "All other parameters are believed to be 'irrelevant' in the sense of the renormalization group" [12]. I am thankful to D.Morrison who pointed me to this paper.

P.S. After this paper had been completed, V.A.Ginzburg gave me the preprint [13] by M.Kontsevich where he also treats rational curves on quintics as solutions to equations induced by the equation of the quintic $X$ in $\mathbb{C} P^{4}$. He finds the numbers $n_{d}$ as Euler numbers of some vector bundles which however live over moduli spaces of stable holomorphic maps to $\mathbb{C} P^{4}$. They differ from our compactified map spaces $\mathbb{C} P^{5 d+4}$ by a way of compactification (which seems to resolve singularities of our compactifying strata). Therefore the 'mirror conjecture' describes how such a resolution should affect our path integral.

\section{References}

[1] P.Candelas, X.C. de la Ossa, P.S.Green, L.Parkes. A pair of Calabi-Yau manifolds as an exactly soluble superconformal theory. — Nucl. Phys. B359 (1991), 21. 
[2] V.V.Batyrev. Dual polyhedra and mirror symmetry for Calabi-Yau hypersurfaces in toric varieties. — Preprint, Essen Univ., 1992.

[3] V.V.Batyrev, D. van Straten. Generalized hypergeometric functions and rational curves on Calabi-Yau complete intersections. — Preprint, Essen Univ., 1993.

[4] M.Atiyah, R.Bott. The moment map and equivariant cohomology. Topology 23 (1984), 1-28.

[5] M.Audin. The topology of torus actions on symplectic manifolds.

Birkhäuser, Basel, 1991.

[6] W.Y.Hsiang. Cohomology theory of topological transformation groups. Springer, N.-Y., 1975.

[7] A.Floer. Symplectic fixed points and holomorphic spheres. - Commun. Math. Phys. 120 (1989), 575-611.

[8] A.Givental. A symplectic fixed point theorem for toric manifolds. Preprint, UC Berkeley, 1992 (to appear in Progr. in Math., Floer's memorial volume).

[9] Y.Ruan, G.Tian. A mathematical theory of quantum cohomology. Preprint, 1994.

[10] A.Givental, B.Kim. Quantum cohomology of flag manifolds and Toda lattices. - Preprint, hep-th/9312096 (to appear in Commun. Math. Phys.

[11] P.Aspinwall, D.Morrison. Topological field theory and rational curves. - Preprint, Oxford, 1991 (to appear in Commun. Math. Phys.)

[12] E.Witten. Phases of $N=2$ theories in two dimensions. - Preprint, IASSNS-HEP-93/3.

[13] M.Kontsevich. Enumeration of rational curves via torus actions. Preprint, 1994.

[14] N.Berline, M.Vergne. Fourier transform of orbits of the coadjoint representation. - In Proceedings of the conference on representation theory 
of reductive groups, (Park City, Utah, 1982), Progress in Math., v. 40, Birkhäuser, Boston, 1983, p. 53-75.

[15] A.Givental. Homological geometry and mirror symmetry. - to appear in Proceedings of the ICM Zürich 1994, Birkhäuser, 1995. 